\title{
Stimulus Frequency Processing in Awake Rat Barrel Cortex
}

\author{
Peter Melzer, Robert N. S. Sachdev, Ned Jenkinson, and Ford F. Ebner \\ Department of Psychology, Vanderbilt University, Nashville, Tennessee 37203
}

In awake rats, we examined the relationship between neural spiking activity in primary somatic sensory cortex and the frequency of whisker stimulation. Neural responses were recorded extracellularly in barrel cortex while single whiskers were deflected with $0.5-18$ air puffs per second (apps), a range that includes the whisk rates observed when rats explore their environment and discriminate surfaces with their whiskers. Twenty-nine neurons in layers III and IV were isolated in three rats ( 23 in barrel columns and 6 in septum columns). At $\leq 9$ apps, cortical neurons responded with one to two spikes per stimulus, whereas at $>9$ apps, the response efficacy was reduced to only 0.2- 0.4 spikes per stimulus. Several mechanisms are discussed that could account for the decrement in responsiveness. Despite this adaptation, neural spike rates increased in direct proportion with stimulus frequency when cast on logarithmic scales. At $>9$ apps, however, this relationship deteriorated in barrel columns in which the response approximately halved. In contrast, septum column cells continued to increase their spike rates linearly up to 18 apps, although they responded at lower magnitude than the barrel column cells. Our findings suggest that septum column neurons are potential candidates to encode stimulus frequency using spike rate across the entire frequency range relevant to rats' whisking behavior.

Key words: depression; excitation; inhibition; vibrissa; rat; somatosensory cortex

\section{Introduction}

Rats use the mystacial whiskers to detect objects and discriminate minute differences in surface texture (Vincent, 1912; GuicRobles et al., 1989; Carvell and Simons, 1990; Brecht et al., 1997; Jenkinson and Glickstein, 2000). During tactile exploration, the animals sweep their whiskers rhythmically at frequencies of 4-15 whisks/s, ramping up to 25 whisks/s just before making a behavioral decision (Welker, 1964; Carvell and Simons, 1990; Mineo et al., 2002; Nguyen et al., 2004; Kleinfeld et al., 2006). Hutson and Masterton (1986) observed that rats can learn to discriminate sinusoidal whisker deflections up to $32 \mathrm{~Hz}$ and proposed that neural processing of whisker touch in rats may be specialized for the extraction of information about texture and form from spike rate-coded whisker oscillations.

The long mystacial whiskers on the snout of the rat are arrayed in five rows that Woolsey and Van der Loos (1970) named A (dorsal) to E (ventral). In each row, four to eight whiskers are numbered beginning with 1 at the caudal end. Four posterior whiskers lie exactly between the rows and are designated by the Greek letters $\alpha$ (dorsal) to $\delta$ (ventral). In layer IV of primary somatic sensory cortex, the whiskers are represented by topographically arranged cytoarchitectonic units that Woolsey and Van der Loos (1970) called barrels. The barrels receive input from the whisker follicles via synaptic transmission from the contralat-

Received June 21, 2006; revised Sept. 21, 2006; accepted 0ct. 11, 2006.

This research was supported by Public Health Service grants (F.F.E.): National Institutes of Health Grant NS25907, J. F. Kennedy Center for Research in Human Development Grant HD-15052, and Vanderbilt Vision Research Center Grant EY-08126. We thank D. Kleinfeld, L. Pagan, B. Williams, J. Long, and M. Walker for their help and M. Armstrong-James for his cogent advice.

Correspondence should be addressed to Peter Melzer, Department of Psychology, Vanderbilt University, 301 Wilson Hall, 111 21st Avenue South, Nashville, TN 37203. E-mail: peter.melzer@vanderbilt.edu.

DOI:10.1523/JNEUROSCI.2620-06.2006

Copyright $\odot 2006$ Society for Neuroscience $\quad 0270-6474 / 06 / 2612198-08 \$ 15.00 / 0$ eral trigeminal nerve to contralateral sensory brainstem nuclei and following the lemniscal pathway through the ipsilateral thalamic ventral posterior medial nucleus (VPM). In cats, trigeminal nerve fibers contacting vibrissal Merkel cells are known to respond to each stimulus up to $1500 \mathrm{~Hz}$ (Gottschaldt and VahleHinz, 1981). Moreover, they follow faithfully, but at lower efficacy, whisker stimulation up to $2800 \mathrm{~Hz}$. In rats, trigeminal ganglion cells respond with high temporal fidelity to sinusoidal stimulation up to $1000 \mathrm{~Hz}$ (Gibson and Welker, 1983; Deschênes et al., 2003; Jones et al., 2006). [Sinusoidal stimuli may specifically affect neural responses and inform the response analyses (Khatri et al., 2004; Mehta and Kleinfeld, 2004). To emphasize this distinction, we express stimulus frequency in hertz only when whiskers were deflected with sinusoidal stimuli.]

In contrast, neural responses in VPM and barrel cortex decrease substantially above 2 whisker deflections/s (Ahissar et al., 2001; Sosnik et al., 2001) (but see Hartings and Simons, 1998). Strong synaptic depression has been observed already at trigeminothalamic endings (Deschênes et al., 2003), and neurons in rat barrel cortex rarely respond to passive whisker stimulation above 40 stimuli/s in anesthetized rats (Simons, 1978; CastroAlamancos, 2004; Khatri et al., 2004; Moore, 2004). In the present study, we used pulsatile whisker deflections produced with 0.5-18 air puffs per second (apps) to specifically examine the effects of stimulus frequency on the spiking activity of neurons in and above the barrels and the tissue between them, i.e., the septa, in alert rats as a complement to a previous study on the processing of stimulus frequency in somatic sensory and motor cortex (Kleinfeld et al., 2002).

\section{Materials and Methods}

Preparation. All procedures were in compliance with National Institutes of Health guidelines, approved by the Vanderbilt University Animal Care 
and Use Committee. The preparation of the animals for recording has been adapted from the method of Nakamura and Ohno (1986) and is similar to those described by Bermejo et al. (1996) and Sachdev et al. (2000). Eight adult male Long-Evans rats [ $\sim 350$ g body weight (b.w.)] were habituated to restraint before single-unit recording. The animals were handled each day beginning near weaning and fed chocolate milk during restraint. The rats that adapted quickly to restraint and drank chocolate milk avidly during the restraint underwent surgery under ketamine (Ketaset, 90 mg/kg b.w., i.m.; Fort Dodge Animal Health, Fort Dodge, IA) and xylazine (Xylazine, $10 \mathrm{mg} / \mathrm{kg}$ b.w., i.m.; Boehringer Ingelheim Vetmedica, St. Joseph, MO) anesthesia to implant a head post over the cerebellum and a recording chamber over barrel cortex. One week after surgery, the rats were habituated to head restraint until they drank chocolate milk consistently again. Habituation to restraint was followed by single-unit recordings.

Cortical recordings. Two miniature screw-advanced drives (Gothard et al., 1996) holding Epoxylite-coated tungsten microelectrodes (2-5 M $\Omega$; Frederic Haer Company, Bowdoinham, ME) were mounted on Teflon caps sealing the recording chamber. The caps were perforated with holes in $5 \times 5,6 \times 6$, or $10 \times 10$ matrices through which the electrodes could be threaded for the transdural introduction into cortex. The microelectrodes were advanced perpendicular to the pial surface until discharges of single units were clearly discernible. Receptive fields were mapped for each location with a hand-held probe. For neurons in barrel columns, we could determine a principal whisker (PW), i.e., the whisker whose deflection evoked the greatest excitatory response at the shortest latency. The deflection of surround whiskers (SW) evoked responses at longer latency and lower magnitude. Septum column cells between barrels often were nearly equally responsive to more than one whisker, so the whisker producing the best response was selected as the PW.

Brief puffs of air from a cone-shaped air hose opening ( $1 \mathrm{~mm}$ diameter) were used to deflect the PW at various frequencies in epochs of $50 \mathrm{~s}$ duration while neural activity was recorded simultaneously from both microelectrodes. Epochs of whisker stimulation were interspersed with $60 \mathrm{~s}$ quiet periods. One investigator constantly monitored the whiskers with a operation microscope to ensure that the air stream moved only the selected whisker during the entire epoch. The data were discarded if there was any detectable movement of other whiskers. After completion of recording with a complete set of stimulus frequencies ranging from 0.5 to 18 apps, the electrodes were advanced until new responsive neurons were isolated and stimulated with the same set of frequencies. At the end of the recording session, three electrolytic lesions ( $5 \mu \mathrm{A}$ for $10 \mathrm{~s}$ ) were placed at the final recording depth and $300 \mu \mathrm{m}$ above and below these sites.

Stimulation. The tip of the air hose was mounted in a goose-neck holder above the stimulated whisker at an angle dorsal and anterior to the animal's head and connected to a solenoid that gated the flow of compressed air (James Long Company, Caroga Lake, NY). Spike 2 software and a 1401 computer interface (Cambridge Electronic Devices, London, UK) were used to trigger the solenoid and provide time stamps for stimulus onset. The air puffs were $30 \mathrm{~ms}$ in duration and adjusted to a force that delivered supramaximal stimulation $(200 \mu \mathrm{m} / \mathrm{s})$. The whisker was deflected with high and low frequencies in alternating sequence, i.e., 18, $0.5,15,1,12,3,9$, and 6 apps, totaling $900,50,750,50,600,150,450$, and 300 stimuli, respectively. Whisker deflection was monitored in three ways: (1) a piezoelectric sensor was placed behind the whisker, ensuring that the pulsatile air flow did not change with changes in stimulus frequency, (2) a force transducer (James Long Company) was used to measure the force of the air puffs at different frequencies, and (3) on select trials, high-speed video images of the whisker movements were acquired (Redlake, Tucson, AZ). The measurements with the piezoelectric sensor and the force transducer both ascertained that the stimulus onset velocity did not vary with increasing stimulus frequency, and the high-speed footage was used to confirm that only the selected whisker was moved.

Data acquisition. The action potentials of single neurons were discriminated on-line with a window discriminator (Bak Electronics, Mount Airy, MD). Action potential duration was measured by cursor displacement across the waveform using a storage oscilloscope (Nicolet 310; LDS, Brighton, MI). Poststimulus time histograms (PSTHs) were constructed on-line with Spike 2 software to ascertain the central receptive field for each neuron using the latency of its discharge.

Histology. After the electrolytic lesions were placed in the electrode tracts at the end of the recording session, the rats were killed with an intraperitoneal injection of pentobarbital ( $80 \mathrm{mg} / \mathrm{kg}$ b.w. Nembutal; Abbott Laboratories, North Chicago, IL) and perfused transcardially with saline, followed by $4 \%$ paraformaldehyde in PBS. The brain was removed and the cortical hemispheres were separated from the brainstem by cutting the fibers of the internal capsule just outside of the thalamus. After hippocampal removal, the hemispheres were flattened and immersed in $30 \%$ sucrose/PBS. Sections, $50 \mu \mathrm{m}$ thick, were cut parallel to the cortical surface on a freezing microtome. The sections were processed for cytochrome oxidase (CO) activity to visualize the barrels in layer IV (WongRiley and Welt, 1980) and to determine whether the electrode penetrations were in a barrel or a septum column and the depth of the recording sites.

Data analyses. Two types of PSTHs were analyzed. In one, PSTHs were constructed from the total number of spikes accumulated during each 50 s epoch of stimulation (called here a "total PSTH"). Alternatively, PSTHs were constructed from the responses to 50 stimuli. In this procedure, only responses that occurred at $\geq 10 \mathrm{~s}$ after the onset of the stimulus epoch were included, limiting the analysis to fully adapted responses (“adapted PSTH”).

Most neurons in rat barrel cortex have finished their short-latency response within $50 \mathrm{~ms}$ after stimulus. Because the interstimulus interval is $55.6 \mathrm{~ms}$ at 18 apps, only spikes that occurred within $50 \mathrm{~ms}$ after the onset of an air puff were used to determine neural spike rate $v$ (spikes/s) and response efficacy $\rho$, i.e., the number of spikes produced per stimulus (spikes/stim). Spontaneous activity was monitored during the $50 \mathrm{~ms}$ before each epoch of whisker stimulation. The levels fluctuated greatly and thus were not subtracted from poststimulus neural responses. Stepwise multiple regression analyses were performed for each neuron to examine the statistical significance of the relationship between response magnitude and stimulus frequency (SPSS, Chicago, IL).

\section{Results}

\section{Methodological considerations}

Spiking activity of single neurons was simultaneously recorded at two separate locations in barrel cortex while a single whisker was stimulated. In a previous study with awake rats in this laboratory (Kleinfeld et al., 2002), we observed small increases in EMG amplitude $(<2 \mu \mathrm{V})$ associated with whisker stimulus frequency, providing indirect evidence that the whiskers were moved concomitantly with the air puffs. However, we cannot exclude the possibility that the air puffs produced additional subtle resonant whisker motion at harmonic frequencies. Although the EMG decreased with increasing frequency, the reduction is not expected to influence the magnitude of neural activation in barrel cortex because, even after facial nerve transection, cortical response magnitudes were not different from those observed in the present study (Sachdev et al., 1999). Because a reduced muscle contraction may have rendered the whiskers slightly more compliant to the air puffs and thus may modify the onset of the neural responses, we refrained from analyzing response latency and stimulus locking.

The location of the recordings varied from animal to animal, as did the PW at each location under these conditions. Establishing the precise depth of the recording sites was complicated by the fact that the microelectrodes needed to penetrate the intact dura. Pushing through the dura may have compressed the underlying cortex so that the advance of the microdrive could not be used to directly measure the depth of the recording sites in barrel cortex. Also, visual inspection of the CO preparations permitted merely a rough estimate of depth because only cortical layer IV with the barrels could be discerned with confidence. Despite these caveats, we could ascertain with the help of the electrolytic lesions that 
approximately half of the neurons examined were situated in layer IV and the other half in deep layer III.

We focus on the results from three of the eight rats used in the present study, because these animals were the most cooperative in providing complete sets of recordings for all stimulus frequencies at complementary locations in barrel cortex. That is, in experiment 1 , the penetrations were in the same barrel column, in experiment 2 in two neighboring barrel columns, and in experiment 3 in a barrel column coupled with the adjacent septum column. We isolated 29 neurons. Their action potentials returned to baseline at $>750 \mu \mathrm{s}$, and, thus, all cells were considered regular-spiking units (Simons, 1978; McCormick et al., 1985). Twenty-three neurons were mapped to barrel columns, and six were located in a septum column. The latter were clearly distinguished in that a PW could not be determined (Armstrong-James and Fox, 1987; Brumberg et al., 1999). Below, we describe the results of each experiment separately and compare the mean responses of the barrel and septum column subpopulations averaged across the three rats with the grand average response of the total population of barrel cortex neurons.

\section{Experiment 1: recordings from a single barrel column}

Figure $1 a$ depicts the barrels in primary somatic sensory cortex reconstructed from $\mathrm{CO}$ preparations. In this experiment, both electrodes traversed the same E2 barrel column (Fig. 1a), and all neurons in those penetrations had contralateral whisker E2 as their PW. Ten cells were isolated along the two tracks, and each responded briskly to deflection of whisker E2 within $\sim 10$ ms of stimulus onset. The data shown for Neuron 1 and Neuron 2 in Figure $1 b-d$ exemplifies the diversity of neural responses at different frequencies in alert animals. The mean responsiveness of all cells in these penetrations across the range of frequencies is shown in Figure 1e. Increasing stimulus frequency decremented the spiking of Neuron 1 and Neuron 2, with the greatest effect at the peak response. Longerlatency components of the response also were reduced at higher frequencies. Despite a remarkable reduction in spiking activity with increasing stimulus frequency, the responses persisted regardless of whether the adapted spiking (Fig. 1b, adapted PSTHs) or total spiking was examined (Fig. $1 c$, total PSTHs). Consistent with the PSTHs, plots of the efficacy of the response $\rho$ (spikes/ stim) against stimulus frequency $f$ revealed two distinct stimulus/ response functions (Fig. 1d). Neuron 1 responded in a nonmonotonic manner with two peaks at $f \leq 9$ apps, whereas $\rho$ of Neuron 2 declined monotonically with increasing stimulus frequency at $f \geq 1$ apps. Despite this difference, both neurons yielded the greatest response efficacy at $f \leq 9$ apps. Neuron 1

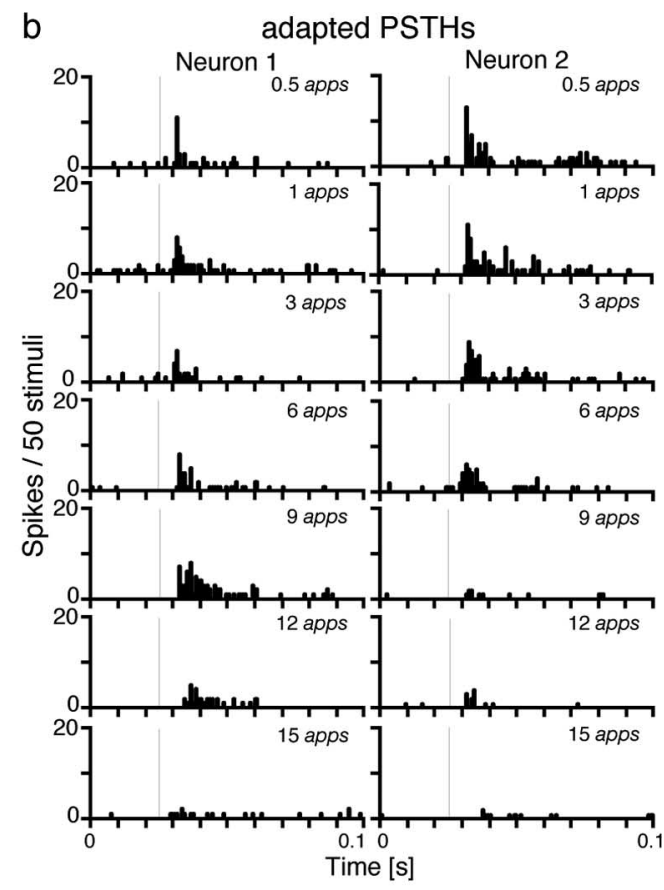

e

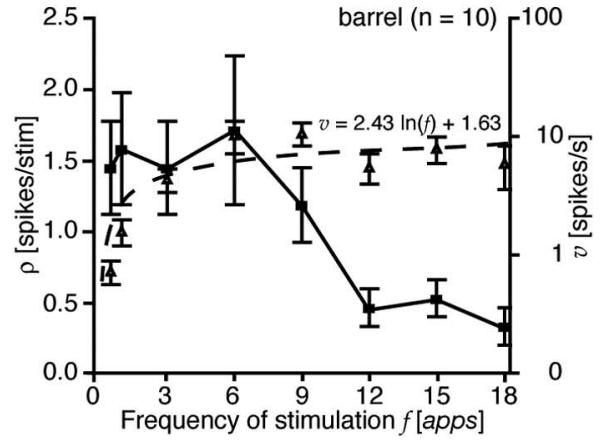

Figure 1. Neural responses in a single barrel column. $\boldsymbol{a}$, A reconstruction of the barrel field from preparations through flattened cortex stained for cytochrome oxidase activity. The letters identify the five rows of barrels (gray) representing the long mystacial briskly at short latency after stimulus onset (thin vertical lines) whether the adapted responses to 50 stimuli (adapted PSTH) (b) or (black squares and line) and Neuron 2 (gray scale), and the spike rate $v$ (triangles; right ordinate, logarithmic scale) matched logarithmic growth (dashed line, equation) (e). The bars through the means represent $\pm 1 S D$.

spiked with greatest $\rho$ (3 spikes/stim) at 9 apps, and Neuron 2 reached its peak (1.5 spikes/stim), already at 1 apps (Fig. $1 d)$. In contrast, above 9 apps, $\rho$ of both neurons was reduced to onefifth of that at 0.5 apps, i.e., the cells produced on average only one spike for every five stimuli presented. This sensory adaptation constituted a common trend among all neurons in this experiment. Figure 1e shows the average response efficacy of the 10 cells plotted on a linear scale against $f$. Mean $\rho$ diminished by two-thirds between 6 and 12 apps. As a consequence, the mean spike rate $v$ of the 10 neurons fitted most closely a logarithmic trend line at $R^{2}=0.53$ when plotted on a logarithmic scale in Figure 1e. Adapted responses were used in this plot. Using the total response in $50 \mathrm{~s}$ epochs did not alter the observed reduction in response, except that an increased number of stimuli lowered SDs. As a consequence, the sensory adaptation attained statistical significance in 8 of the 10 cells $(p \leq 0.05)$. 

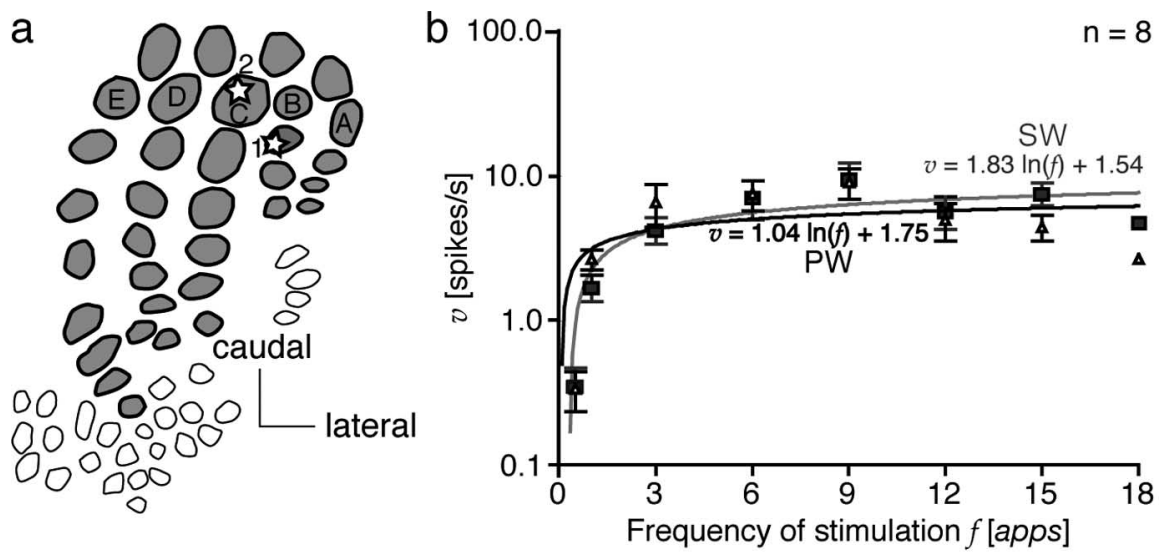

C
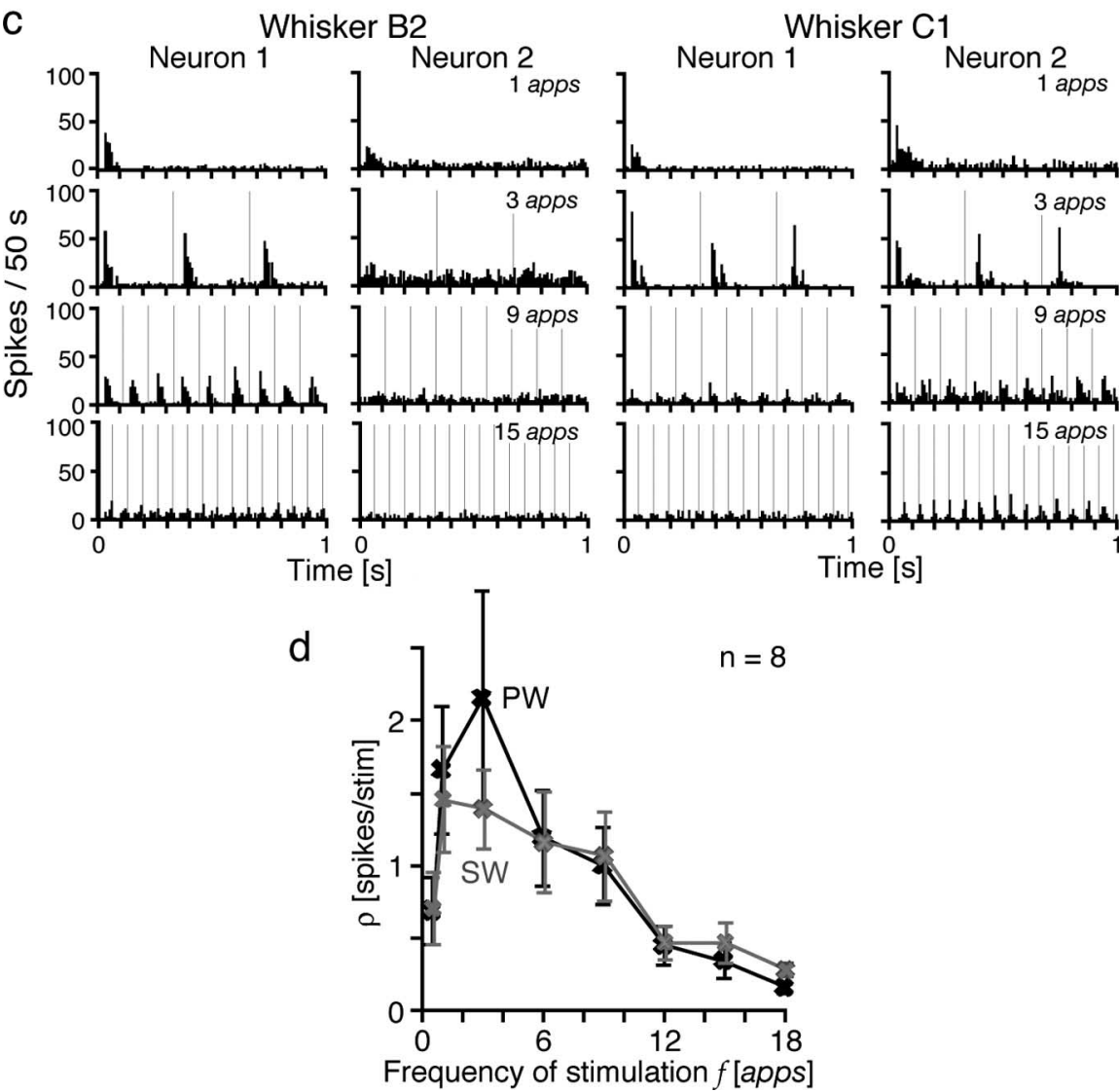

Figure 2. Neural responses in adjacent barrel columns. $\boldsymbol{a}$, Electrode 1 (star 1 ) was positioned in the column of barrel B2, and electrode 2 (star 2) was positioned in the column of barrel $\mathbf{C} 1 . \boldsymbol{b}$, The mean spike rates $v$ for the eight cells examined were plotted logarithmically against stimulus frequency $f$. Means, trend lines, and their equations are shown for responses to the deflection of whisker B1 or C1 as the PW (triangles, black line) or the SW (squares, gray line). c, Total PSTHs of exemplary neural responses are shown for each barrel column. The vertical thin lines indicate the onset of the whisker deflection. $\boldsymbol{d}, \mathrm{PW}$ (black) deflection yielded a higher response efficacy $\rho$ at peak than SW (gray) deflection. The bars through the means represent \pm 1 SD.

tions between 12 and 18 apps to such a degree that the fit was distinctly lower $\left(R^{2}=0.23\right)$ than that produced with SW deflection $\left(R^{2}=0.65\right)$.

The PSTHs in Figure $2 c$ show examples for typical responses of the cells recorded in barrel columns B2 (Neuron 1) and C1 (Neuron 2) at increasing $f$. Although neural responses appeared locked to the stimulus across the entire frequency range, the cells responded more vigorously to deflection of their respective PW than to deflection of their SW. That is, deflection of whisker B2 evoked more spikes in contralateral barrel column B2 (Neuron 1) than deflection of whisker C1. Conversely, deflection of whisker $\mathrm{C} 1$ commonly produced more spikes in contralateral barrel column C1 (Neuron 2) than deflection of whisker B2. As a consequence, $\rho$ at $f \leq 3$ apps was on average 1.4 times greater with PW deflection than with SW deflection (Fig. $2 d$ ). At $f \geq 6$ apps, however, the neural response efficacy for either whisker diminished with similar slopes, leveling off at a slightly higher magnitude for SW deflection. The inverse relationship between $\rho$ and $f$ proved statistically significant for seven of the eight cells when the PW was deflected and for five of eight cells when the SW was deflected.

\section{Experiment 3: recordings from nearby barrel and septum columns}

In this condition, electrode 1 was located in a septum column, and electrode 2 was located in the adjacent $\mathrm{C} 4$ barrel column (Fig. 3a). Eleven neurons were recorded in these penetrations: six in the septum column and five in the barrel column. Deflection of contralateral whisker $\mathrm{C} 4$ evoked responses at both locations. In Figure 3, $b$ and $c$, the mean neural spike rate $v$ is plotted logarithmically, and the mean response efficacy $\rho$ is plotted linearly against stimulus frequency $f$. For barrel column cells (Fig. $3 b$ ), $v$ increased with increasing $f$ to peak at 9 apps, considerably exceeding the values predicted by the logarithmic trend line. At greater $f$, however, $v$ approximately halved, resulting in the greatest negative deviation from the predictions.

Experiment 2: recordings from neighboring barrel columns In this experiment, the electrodes traversed through barrel columns B2 and C1 (Fig. 2a). Eight neurons were isolated, having whiskers B2 and C1 either as a PW or an SW. The effect of stimulus frequency $f$ on the mean neural spike rate $v$ was similar to that observed in the first experiment (compare Figs. $2 b, 1 e$ ). Deflection of either the PW or the SW increased $v$ with increasing $f$ logarithmically (Fig. 2b). That is, PW and SW deflection increased $v$ rapidly at $f \leq 3$ apps, beyond which $v$ approached a plateau. However, the PW response overshot the values predicted by the trend line between 3 and 9 apps and undershot the predic-
As a consequence, $v$ fitted with the lowest coefficient observed in the present study, i.e., $R^{2}=0.13$. Consistent with this finding, neural response efficacy in the barrel column peaked at $\sim 1.5$ spikes/stim at 1 apps and then declined to merely $\sim 0.05$ spikes/ stim at 18 apps (Fig. $3 b$ ). In striking contrast, for the septum column cells (Fig. $3 c$ ), $v$ fitted to the trend line with the highest coefficient observed in the present study, i.e., $R^{2}=0.94$, continuing to increase even up to 18 apps. The response efficacy of the septum column cells peaked at approximately half the spikes/ stim reached by the barrel column neurons but attained a steady state at $f>6$ apps. As a result, at 18 apps the septum column cells 

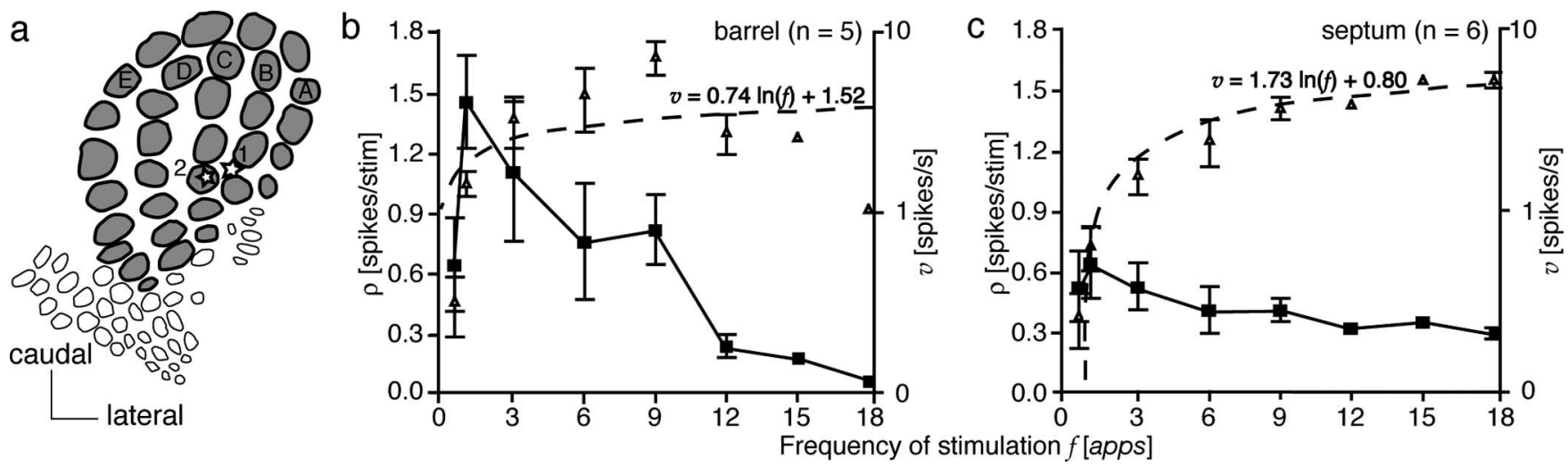

Figure 3. Neural responses in nearby barrel and septum columns. Electrode 1 (star 1) was positioned in the column of the septum between barrels B3, B4, C3, and C4. Electrode 2 (star 2) was positioned in the column of barrel $C 4(\boldsymbol{a})$. Deflection of whisker $C 4$ increased neural activity in both locations. The mean spike rate $v$ of the five cells in the barrel column (triangles, logarithmic ordinate) increased with increasing stimulus frequency $f$ up to 9 apps $(\boldsymbol{b})$, beyond which it approximately halved, resulting in the greatest disparity with the logarithmic trend line (dashed line, equation). The mean response efficacy $\rho$ (squares, linear ordinate) peaked at 1 apps and then declined to the lowest level observed in the present study at 18 apps. In contrast, the mean $v$ of the six septum column neurons increased in good correspondence with logarithmic growth ( $\boldsymbol{c}$; same conventions as in $\boldsymbol{b}$ ). Their response efficacy peaked at 1 apps, decreased less rapidly with increasing $f$ than in the barrel columns, and approached a steady state at frequencies $\geq 6$ apps. The bars through the means represent \pm 1 SD.

(Fig. 3c) produced approximately six times as many spikes/stim as the barrel column cells (Fig. $3 b$ ). Consistent with this difference, the inverse relationship between $\rho$ and $f$ was statistically significant for four of the five barrel column cells but only three of the six septum column cells.

\section{Population response in barrel cortex}

In Figure 4, we compare the grand average response to frequency of whisker stimulation of the total population of 29 cells isolated in the three rats with that of the barrel column neurons and that of the septum column neurons. The prevalence of the six septum column neurons in the total population, i.e., $20 \%$, is realistic in caudal rat barrel cortex assuming an average barrel diameter of $400 \mu \mathrm{m}$ and an average septum width of $80 \mu \mathrm{m}$.

In Figure $4 a$, the mean response efficacy $\rho$ is plotted linearly against stimulus frequency $f$ for the three samples. For both barrel and septum column cells, $\rho$ peaked at 1 apps, declining approximately monotonically with additional increases in $f$. However, $\rho$ in barrel columns was threefold greater at peak than that in septum columns and decreased at greater slope with increasing $f$, eventually falling to one-fifth of the peak response. The different slopes of decline resulted in the convergence of $\rho$ at $f \geq 12$ apps. Below 12 apps, the greater prevalence and the greater change in response efficacy of the barrel column cells predominantly influenced the dynamics of the response of the total population, bringing the average neural response in barrel cortex closer to the barrel column response.

Figure $4 b$ shows that averaging spike rate $v$ across the three experiments of the present study did not improve the fit to logarithmic growth for barrel column neurons $\left(R^{2}=0.37\right)$. In contrast, the relationship between $v$ and $f$ of septum column neurons was of such greater tightness $\left(R^{2}=0.94\right)$ that, despite their lower spike rate and prevalence, septum column neurons noticeably improved the fit of the grand average to logarithmic growth for the whole population $\left(R^{2}=0.59\right)$, exceeding 1.6 times the fit for barrel column neurons alone.

Figure $4 c$ demonstrates the fundamental difference in spike rate growth between barrel column and septum column neurons most clearly. Here, $v$ and $f$ are both plotted on logarithmic axes. The spike rate in barrel columns increases nearly in direct proportion with $f$ up to 9 apps but is profoundly reduced at greater stimulus frequencies. The growth of $v$ for the total population parallels this trend. In contrast, although septum column cells responded at lower $v$ than barrel column cells at $f \leq 9$ apps, persistent linear growth ensued up to 18 apps, ultimately matching the reduced $v$ in barrel columns (note the nearly overlapping means at $f \geq 12$ apps in Fig. $4 c$ ). This striking linearity of response suggests that septum column cells constitute a unique subset of neurons in barrel cortex capable of encoding the frequency of whisker stimulation by proportionate spiking across the whole spectrum of frequencies tested (0.5-18 apps).

\section{Discussion}

We examined effects of the frequency of whisker stimulation on neural responses in awake rat barrel cortex. The animals were trained extensively with rewards to sit quietly under restraint such that single-unit activity could be recorded simultaneously from two separate cortical locations while single whiskers were deflected with air puffs. Whisker deflection evoked robust neural responses in barrel cortex. Response efficacy $\rho$ and spike rate $v$ changed substantially with stimulus frequency in and above the barrels and the septa between them. Possible mechanisms underlying the neural responses are discussed below.

\section{Response efficacy}

The peak response efficacy averaged across all neurons isolated in barrel cortex is in good agreement with the data reported in other studies with awake rats (Castro-Alamancos, 2004; Krupa et al., 2004). The decrease in efficacy of the neural response with increasing stimulus frequency is consistent with numerous findings in anesthetized rats (Simons, 1978; Fanselow and Nicolelis, 1999; Ahissar et al., 2001; Chung et al., 2002; Kleinfeld et al., 2002; Garabedian et al., 2003; Castro-Alamancos, 2004; Khatri et al., 2004; Higley and Contreras, 2006; Melzer et al., 2006). The underlying mechanisms may involve corticocortical and/or corticosubcortical interactions.

\section{Corticocortical interactions}

The diminution in response efficacy observed in the present study may simply be the result of the high rates of sustained stimulation producing cumulative increases in inhibition within intracortical circuits. A role for inhibition in barrel columns is supported by the finding that deflection of the same whisker twice evokes a weaker response if the second stimulus is presented 

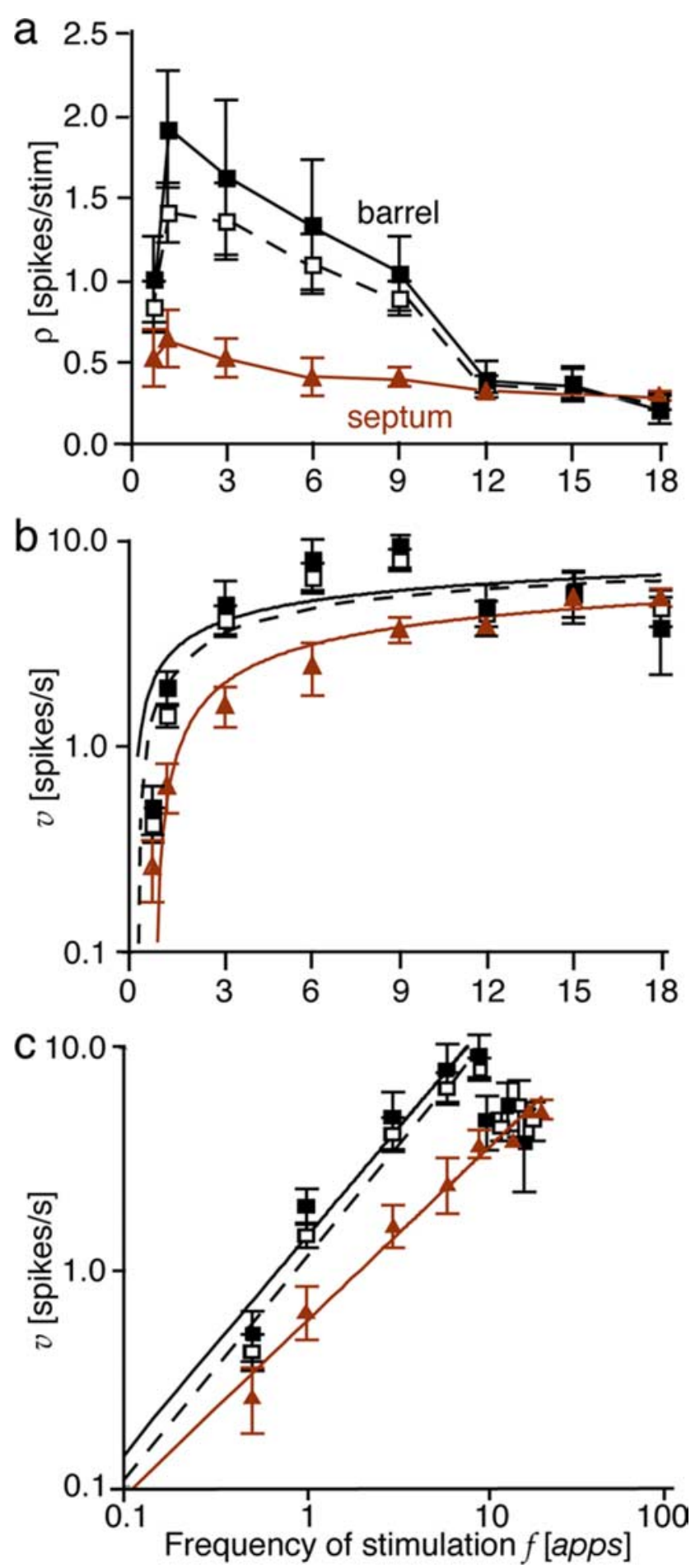

Figure 4. Grand average population responses. Response efficacy $\rho$ and spike rate $v$ are plotted against stimulus frequency $f$ for the 23 barrel column neurons (filled squares and black lines), the six septum column neurons (brown triangles and lines), and the total population of barrel cortex neurons (unfilled squares and dashed lines). $\rho$ peaked at 1 apps and then declined continuously $(\boldsymbol{a})$, although the neural spike rate $v$ tended to grow logarithmically with increasing $f(\boldsymbol{b})$. However, plotting $v$ and $f$ logarithmically reveals that septum column neurons achieve linear growth up to 18 apps (c). The values at $f>9$ apps were slightly displaced to dissociate the symbols. The bars through the means represent \pm 1 SD.

within 20-100 ms (Simons, 1985). It was suggested that this feedforward, within-barrel column inhibition leads to the short, stimulus-locked volleys of neural activity (Simons, 1978; Ito, 1985; Armstrong-James and Fox, 1987) that resemble the peri- odic spiking Mountcastle et al. (1969) observed in the macaque primary somatic sensory cortex with vibrotactile stimulation of the glabrous skin. These authors noted that the periodicity of neural discharge closely matched the interstimulus interval and concluded that the frequency of stimulation is most likely directly encoded in the temporal sequence of the action potentials.

Under this hypothesis, the resolution of high stimulus frequencies in a temporal code requires coincidence detection at high resolution. Fulfilling this requirement, disynaptic intracortical inhibition appears to balance neural excitation, narrowing the window for spike output to $\sim 2-10 \mathrm{~ms}$ (Agmon and Connors, 1992; Swadlow and Gusev, 2000; Porter et al., 2001; Gabernet et al., 2005; Higley and Contreras, 2006). Alternatively, highfrequency stimulation of cortical neurons in brain slices has been shown to depress excitatory corticocortical synapses more than inhibitory synapses (Galarreta and Hestrin, 1998; Varela et al., 1999), and a reduction in amplitude of EPSPs may ultimately enhance the effect of inhibition underlying the sensory adaptation we observed. However, our findings suggest that, in awake rat barrel cortex, the balance between excitation and inhibition may be shifted toward excitation, because septum column neurons succeeded in keeping their response efficacy steady at high stimulus frequencies.

\section{Cortico-subcortical interactions}

In addition to intracortical inhibition, the observed sensory adaptation may be in part the result of suppression at peripheral or subcortical synapses, because the peak response at short latency, i.e., the response to thalamocortical input, was considerably diminished at stimulus frequencies $>9$ apps. Neurons in the somatic sensory thalamus but not in the brainstem (Sosnik et al., 2001) show decrements in response similar to cortical neurons (Ahissar et al., 2001), suggesting a partially thalamic origin of sensory adaptation. Consistent with this contention, stimulation of thalamocortical afferents produced a greater response in barrel neurons than stimulation of intracortical afferents (Gil et al., 1999). Furthermore, the frequency of stimulation of intracortical inputs needed to be four times greater than that of thalamocortical inputs to suppress barrel neuron excitability (Contreras and Llinas, 2001). Therefore, as an alternative to intracortical inhibition, thalamocortical afferents may be depressed presynaptically (Chung et al., 2002) via inhibitory corticothalamic feedback (Ahissar et al., 2000), leading to the diminution of input effect that Higley and Contreras (2005) describe as "disfacilitation."

Because the thalamic response to brainstem input is influenced by cortical activity (Diamond et al., 1992a; Ghazanfar et al., 2001; Li and Ebner, 2006), corticothalamic feedback may dynamically modulate the balance between excitation and inhibition in barrel cortex regulating neural responsiveness commensurate with stimulus frequency. Septum cells receive tactile input via a pathway separate from the lemniscal route to barrel neurons. This paralemniscal pathway originates in the spinal trigeminal sensory brainstem nucleus and projects to cortex via the medial division of the thalamic posterior nucleus (POm) ( $\mathrm{Lu}$ and Lin, 1993; Kim and Ebner, 1999). Diamond et al. (1992b) observed a stimulus frequency-related decrease in neural response efficacy in POm similar to our finding in the septum column. However, the rats were anesthetized. At 10 stimuli/s, the response efficacy decreased to half that of the septum column cells in the present study, and responses to higher frequencies were not examined.

The persistent response efficacy of septum column neurons we observed in the awake animals may be based on the peculiarity that neural responses in POm are constantly inhibited by affer- 
ents from the zona incerta (Deschênes et al., 2005). This inhibition is modulated by cholinergic brainstem input related to arousal (Trageser et al., 2006). Cholinergic input to motor cortex facilitates the whisker movements found in awake rats (Berg et al., 2005). Therefore, POm neurons may be less inhibited when the animals are alert, and excitatory thalamocortical feedback may gain strength with increasing stimulus frequency (Deschênes et al., 2005).

\section{Spike rate}

Our second observation of note is that neural spike rates in awake rat barrel cortex were linearly related to the frequency of whisker stimulation. That is, spike rates increased in proportion with stimulus frequency up to 9 apps, and this proportionality was maintained whether the PW or the SW was deflected. Although greater than in the septum column at stimulus frequencies $\leq 9$ apps, spike rates in the barrel columns did not sustain linear growth at frequencies $>9$ apps, perhaps because of a subtle transformation of the whisker deflection from completely reset to baseline to a stimulus resembling a ramp-and-hold deflection (Melzer et al., 2006). Remarkably, the septum column neuron responses proved steadfast, continuing to increase in rate up to the highest stimulus frequency tested. Hence, our findings provide support for the idea that the frequency of whisker stimulation may be encoded in neural spike rate in barrel columns up to 9 apps and in septum columns up to 18 apps. In accord, recent studies in macaques performing vibrotactile discrimination tasks demonstrate that spike rate may suffice to encode stimulus frequency in primate somatic sensory cortex (Luna et al., 2005).

Consistent with the sustained responsiveness of septum column neurons, SW deflection produced higher spike rates in barrel column neurons than PW deflection at stimulus frequencies $\geq 15$ apps. Surround receptive fields of barrel cells depend on input from adjacent barrels (Armstrong-James et al., 1991, 1992; Fox et al., 2003), and, at high stimulus frequencies, the barrel column response may be augmented by a more active septum input. Monotonically rising stimulus/response functions appear robust in septum columns, because such functions were observed even in anesthetized rats and with multi-whisker stimulation (Melzer et al., 2006). Septum column neurons may, therefore, constitute a class of cells well suited to process frequencies of whisker stimulation in an integrate-and-fire mode (Shadlen and Newsome, 1998) in a range in which rats are known to whisk during exploration and tactile discrimination (Welker, 1964; Carvell and Simons, 1995; Fee et al., 1997; Gao et al., 2001; Mineo et al., 2002; Berg and Kleinfeld, 2003).

In analogy to the greater sensitivity to high contrast of primate visual cortex neurons receiving parvocellular input compared with those receiving magnocellular input (Allison et al., 2000), septum column neurons may exert greater gain control at high frequencies of whisker stimulation than barrel column cells, and the different origins of tactile input may explain the observed differences in range of linear responsiveness. Yu et al. (2006) report that lemniscal VPM neurons respond when a moving whisker hits an obstacle, whereas paralemniscal POm neurons respond only to the unimpeded whisker movement. The authors suggest that the lemniscal pathway may be involved in object detection, whereas the paralemniscal pathway may be engaged in the processing of the fundamental beat of whisking. Other recent studies indicate that spike rates in rat barrel cortex may increase proportionately with the frequency of vibrotactile stimulation up to $400 \mathrm{~Hz}$ (Andermann et al., 2004; Arabzadeh et al., 2005; Kleinfeld et al., 2006). Separate channels processing vibrotactile stim- uli in distinct but overlapping frequency bands have been described for the primate somatic sensory system (Bolanowski et al., 1988). Perhaps, in rats, sinusoidal vibratory whisker stimuli are processed in channels different from those that process whisker deflections, and septum cells may specifically contribute to the processing of whisker oscillations.

\section{References}

Agmon A, Connors BW (1992) Correlation between intrinsic firing patterns and thalamocortical synaptic responses of neurons in mouse barrel cortex. J Neurosci 12:319-329.

Ahissar E, Sosnik R, Haidarliu S (2000) Transformation from temporal to rate coding in a somatosensory thalamocortical pathway. Nature 406:302-306.

Ahissar E, Sosnik R, Bagdasarian K, Haidarliu S (2001) Temporal frequency of whisker movement. II. Laminar organization of cortical representation. J Neurophysiol 86:354-367.

Allison JD, Melzer P, Ding Y, Bonds AB, Casagrande VA (2000) Differential contributions of magnocellular and parvocellular pathways to the contrast response of neurons in bush baby primary visual cortex (V1). Vis Neurosci 17:71-76.

Andermann ML, Ritt J, Neimark MA, Moore CI (2004) Neural correlates of vibrissa resonance; band-pass and somatotopic representation of highfrequency stimuli. Neuron 13:451-463.

Arabzadeh E, Zorzin E, Diamond ME (2005) Neural encoding of texture in the whisker sensory pathway. PloS Biol 3:e17.

Armstrong-James M, Fox K (1987) Spatiotemporal convergence and divergence in the rat SI "barrel" cortex. J Comp Neurol 263:265-281.

Armstrong-James M, Callahan CA, Friedman MA (1991) Thalamo-cortical processing of vibrissal information in the rat. I. Intracortical origins of surround but not centre-receptive fields of layer IV neurones in the rat S1 barrel field cortex. J Comp Neurol 303:193-210.

Armstrong-James M, Fox K, Das-Gupta A (1992) Flow of excitation within rat barrel cortex on striking a single vibrissa. J Neurophysiol 68:1345-1358.

Berg RW, Kleinfeld D (2003) Rhythmic whisking by rat: retraction as well as protraction of the vibrissae is under active muscular control. J Neurophysiol 89:104-117.

Berg RW, Friedman B, Schroeder LF, Kleinfeld D (2005) Activation of nucleus basalis facilitates cortical control of a brain stem motor program. J Neurophysiol 94:699-711.

Bermejo R, Harvey M, Gao P, Zeigler HP (1996) Conditioned whisking in the rat. Somatosens Mot Res 13:225-233.

Bolanowski Jr SJ, Gescheider GA, Verrillo RT, Checkosky CM (1988) Four channels mediate the mechanical aspects of touch. J Acoust Soc Am 84:1680-1694

Brecht M, Preilowski B, Merzenich MM (1997) Functional architecture of the mystacial vibrissae. Behav Brain Res 84:81-97.

Brumberg JC, Pinto DJ, Simons DJ (1999) Cortical columnar processing in the rat whisker-to-barrel system. J Neurophysiol 82:1808-1817.

Carvell GE, Simons DJ (1990) Biometric analyses of vibrissal tactile discrimination in the rat. J Neurosci 10:2638-2648.

Carvell GE, Simons DJ (1995) Task and subject related differences in sensorimotor behavior during active touch. Somatosens Mot Res 12:1-9.

Castro-Alamancos MA (2004) Absence of rapid sensory adaptation in neocortex during information processing states. Neuron 41:455-464.

Chung S, Li X, Nelson SB (2002) Short-term depression at thalamocortical synapses contributes to rapid adaptation of cortical sensory responses in vivo. Neuron 34:437-446.

Contreras D, Llinas R (2001) Voltage sensitive dye imaging of neocortical spatiotemporal dynamics to afferent activation frequency. J Neurosci 21:9403-9413.

Deschênes M, Timofeeva E, Lavallée P (2003) The relay of high-frequency sensory signals in the whisker-to-barreloid pathway. J Neurosci 23:6778-6787.

Deschênes M, Timofeeva E, Lavallée P, Dufresne C (2005) The vibrissal system as a model of thalamic operations. Prog Brain Res 149:31-40.

Diamond ME, Armstrong-James M, Ebner FF (1992a) Somatic sensory responses in the rostral sector of the posterior group (POm) and in the ventral posterior medial nucleus (VPM) of the rat thalamus. J Comp Neurol 318:462-476.

Diamond ME, Armstrong-James M, Budway MJ, Ebner FF (1992b) Somatic 
sensory responses in the rostral sector of the posterior group (POm) and in the ventral posterior medial nucleus (VPM) of the rat thalamus: dependence on the barrel field cortex. J Comp Neurol 319:66-84.

Fanselow EE, Nicolelis MAL (1999) Behavioral modulation of tactile responses in the rat somatosensory system. J Neurophysiol 19:7603-7616.

Fee MS, Mitra PP, Kleinfeld D (1997) Central versus peripheral determinants of patterned spike activity in rat vibrissa cortex during whisking. J Neurophysiol 78:1144-1149.

Fox K, Wright N, Wallace H, Glazewski S (2003) The origin of cortical surround receptive fields studied in the barrel cortex. J Neurosci 23:8380-8391.

Gabernet L, Jadhav SP, Feldman DE, Carandini M, Scanziani M (2005) Somatosensory integration controlled by dynamic thalamocortical feedforward inhibition. Neuron 48:315-327.

Galarreta M, Hestrin S (1998) Frequency-dependent synaptic depression and the balance of excitation and inhibition in the neocortex. Nat Neurosci 1:587-593.

Gao P, Bermejo R, Zeigler HP (2001) Whisker deafferentation and rodent whisking patterns: behavioral evidence for a central pattern generator. J Neurosci 21:5374-5380.

Garabedian CE, Jones SR, Merzenich MM, Dale A, Moore CI (2003) Bandpass response properties of rat SI neurons. J Neurophysiol 90:1379-1391.

Ghazanfar AA, Krupa DJ, Nicolelis MA (2001) Role of cortical feedback in the receptive field structure and nonlinear response properties of somatosensory thalamic neurons. Exp Brain Res 141:88-100.

Gibson JM, Welker W (1983) Quantitative studies of stimulus coding in first-order vibrissa afferents of rats. 2. Adaptation and coding of stimulus parameters. Somatosens Res 1:95-117.

Gil Z, Connors BW, Amitai Y (1999) Efficacy of thalamocortical and intracortical synaptic connections: quanta, innervation, and reliability. Neuron 23:385-397.

Gothard KM, Skaggs WE, Moore KM, McNaughton BL (1996) Binding of hippocampal CA1 neural activity to multiple reference frames in a landmark-based navigation task. J Neurosci 16:823-835.

Gottschaldt KM, Vahle-Hinz C (1981) Merkel cell receptors: structure and transducer function. Science 214:183-186.

Guic-Robles E, Valdiviesco C, Guajardo G (1989) Rats can learn a roughness discrimination task using only their vibrissal system. Behav Brain Res 31:285-289.

Hartings JA, Simons DJ (1998) Thalamic relay of afferent responses to 1- to $12-\mathrm{Hz}$ whisker stimulation in the rat. J Neurophysiol 80:1016-1019.

Higley MJ, Contreras D (2005) Integration of synaptic responses to neighboring whiskers in rat barrel cortex in vivo. J Neurophysiol 93:1920-1934.

Higley MJ, Contreras D (2006) Balanced excitation and inhibition determine spike timing during frequency adaptation. J Neurosci 26:448-457.

Hutson K, Masterton RB (1986) The sensory contribution of a single vibrissa's cortical barrel. J Neurophysiol 56:1196-1223.

Ito M (1985) Processing of vibrissa sensory information within rat neocortex. J Neurophysiol 54:479-490.

Jenkinson EW, Glickstein M (2000) Whiskers, barrels, and cortical efferent pathways in gap crossing by rats. J Neurophysiol 84:1781-1789.

Jones LM, Kwegyir-Afful E, Keller A (2006) Whisker primary afferents encode temporal frequency of moving gratings. Somatosens Mot Res 23:45-54.

Khatri V, Hartings JA, Simons DJ (2004) Adaptation in thalamic barreloid and cortical barrel neurons to periodic whisker deflections varying in frequency and velocity. J Neurophysiol 92:3244-3254.

Kim U, Ebner FF (1999) Barrels and septa: separate circuits in rat barrel field cortex. J Comp Neurol 408:489-505.

Kleinfeld D, Sachdev RN, Merchant LM, Jarvis MR, Ebner FF (2002) Adaptive filtering of vibrissa input in motor cortex of rat. Neuron 34:1021-1034.

Kleinfeld D, Ahissar E, Diamond ME (2006) Active sensation: insights from the rodent vibrissa sensorimotor system. Curr Opin Neurobiol 16:1-10.

Krupa DJ, Wiest MC, Shuler MG, Laubach M, Nicolelis MA (2004) Layerspecific somatosensory cortical activation during active tactile discrimination. Science 304:1989-1992.

Luna R, Hernandez A, Brody CD, Romo R (2005) Neural codes for percep- tual discrimination in primary somatosensory cortex. Nat Neurosci 8:1210-1219.

McCormick DA, Connors BW, Lighthall JW, Prince DA (1985) Comparative electrophysiology of pyramidal and sparsely spiny stellate neurons of the neocortex. J Neurophysiol 54:782-806.

Li L, Ebner FF (2006) Cortical modulation of spatial and angular tuning maps in the rat thalamus. J Neurosci, in press.

Lu SM, Lin RC (1993) Thalamic afferents of the barrel cortex: a light- and electron-microscopic study using Phaseolus vulgaris leucoagglutinin as an anterograde tracer. Somatosens Mot Res 10:1-16.

Mehta SB, Kleinfeld D (2004) Patterned sensory input in the rat vibrissa system. Neuron 41:181-184.

Melzer P, Champney GC, Maguire MJ, Ebner FF (2006) Rate code and temporal code for frequency of whisker stimulation in rat primary and secondary somatic sensory cortex. Exp Brain Res 172:370-386.

Mineo L, Melzer P, Ebner F (2002) Whisk rate of blind rats is accelerated during object discrimination. Soc Neurosci Abstr 28:257.9.

Moore CI (2004) Frequency-dependent processing in the vibrissa sensory system. J Neurophysiol 91:2390-2399.

Mountcastle VB, Talbot WH, Sakata H, Hyvärinen J (1969) Cortical neuronal mechanisms in flutter-vibration studied in unanesthetized monkeys. Neuronal periodicity and frequency discrimination. J Neurophysiol 32:453-484.

Nakamura K, Ohno T (1986) Lateral hypothalamus neuron involvement in integration of natural and artificial rewards and cue signals. J Neurophysiol 55:163-181.

Nguyen QT, Wessel R, Kleinfeld D (2004) Developmental regulation of active and passive membrane properties in rat vibrissae motor neurons. J Physiol (Lond) 556:203-219.

Porter JT, Johnson CK, Agmon A (2001) Diverse types of interneurons generate thalamus-evoked feedforward inhibition in the mouse barrel cortex. J Neurosci 21:2699-2710.

Sachdev RNS, Melzer P, Jenkinson E, Ebner FF (1999) Dual electrode recording from the awake rat S1 barrel cortex. Somatosens Mot Res 16:165-166.

Sachdev RNS, Sellien H, Ebner FF (2000) Direct Inhibition evoked by whisker stimulation in somatic sensory (S1) barrel field cortex of the awake rat. J Neurophysiol 84:1497-1504.

Shadlen MN, Newsome WT (1998) The variable discharge of cortical neurons: implications for connectivity, computation, and information coding. J Neurosci 18:3870-3896.

Simons DJ (1978) Response properties of vibrissa units in rats SI somatosensory neocortex. J Neurophysiol 41:798-820.

Simons DJ (1985) Temporal and spatial integration in the rat S1 vibrissa cortex. J Neurophysiol 54:615-635.

Sosnik R, Haiderliu S, Ahissar E (2001) Temporal frequency of whisker movement. I. Representations in brain stem and thalamus. J Neurophysiol 86:339-353.

Swadlow HA, Gusev AG (2000) The influence of single VB thalamocortical impulses on barrel columns of rabbit somatosensory cortex. J Neurophysiol 83:2802-2813.

Trageser JC, Burke KA, Masri R, Li Y, Sellers L, Keller A (2006) Statedependent gating of sensory inputs to zona incerta. J Neurophysiol 96:1456-1463.

Varela J, Song S, Turrigiano G, Nelson S (1999) Differential depression at excitatory and inhibitory synapses in visual cortex. J Neurosci 19:4293-4304.

Vincent SB (1912) The function of the vibrissa in the behavior of the white rat. Behav Monogr 1:7-86.

Welker WI (1964) Analysis of sniffing of the albino rat. Behav 22:223-244.

Wong-Riley MT, Welt C (1980) Histochemical changes in cytochrome oxidase of cortical barrels after vibrissal removal in neonatal and adult mice. Proc Natl Acad Sci USA 77:2333-2337.

Woolsey TA, Van der Loos H (1970) The structural organization of layer IV in the somatosensory region (SI) of mouse cerebral cortex. The description of a cortical field composed of discrete cytoarchitectonic units. Brain Res 17:205-242.

Yu C, Derdikman D, Haidarliu S, Ahissar E (2006) Parallel thalamic pathways for whisking and touch signals in the rat. PloS Biol 4:e124. 